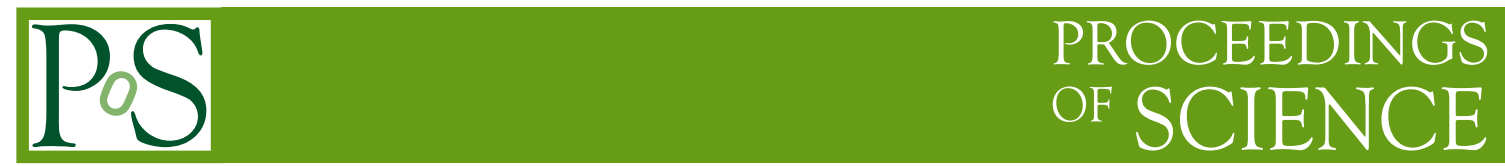

\title{
The European Pulsar Timing Array
}

\section{Roy Smits*}

The University of Manchester, School of Physics and Astronomy, Jodrell Bank Netherlands Institute for Radio Astronomy (ASTRON), Dwingeloo, The Netherlands E-mail: rsmits@jb.man.ac.uk

\section{The EPTA collaboration}

http://www.epta.eu.org

The European Pulsar Timing Array (EPTA) is a multi-institutional, multi-telescope collaboration, with the goal of using high-precision pulsar timing to directly detect gravitational waves. We discuss the EPTA member telescopes, current efforts and the Large European Array for Pulsars, in which the five major European telescopes involved in pulsar timing will be combined to provide a coherent array that will give similar sensitivity to the Arecibo radio telescope, and larger sky coverage.

ISKAF2010 Science Meeting - ISKAF2010

June 10-14, 2010

Assen, the Netherlands

${ }^{*}$ Speaker. 


\section{Introduction}

Pulsars are highly magnetized neutron stars (NS), the remnants of the supernovae of massive stellar progenitors (Gold 1968; Pacini 1968). The majority of known pulsars rotate approximately once every second, and most are visible via their radio emission. It is generally accepted that radio pulsars radiate from above their magnetic poles; with each rotation of the NS, we observe a cross-section through the emission region that cuts our line of sight at Earth (Gold 1968; Pacini 1968; Goldreich \& Julian 1969; Sturrock 1971). This gives rise to the "pulses" that lend pulsars their name. The intrinsic periodicity of most pulsars is remarkably stable, with typical period derivatives of $\sim 10^{-15} \mathrm{~s} \mathrm{~s}^{-1}$ (see e.g., Lorimer, D. R. and Kramer, M. 2005).

There is a population of so-called "recycled" pulsars with rotational periods of $\sim 1-100$ ms. They are the result of a mass-accretion episode from a stellar binary companion onto the NS. The accreted matter carries angular momentum, resulting in the "spin-up" of the NS (Smarr \& Blandford 1976; Alpar et al. 1982; Radhakrishnan \& Srinivasan 1982; Srinivasan \& van den Heuvel 1982). These millisecond pulsars (MSPs) are observed to be more stable as a population than the mainstream pulsars, with rotational period derivatives of $\sim 10^{-20} \mathrm{~s} \mathrm{~s}^{-1}$ (see e.g., Lorimer, D. R. and Kramer, M. 2005). They also show fewer instances of intrinsic, often low-frequency noise, corresponding to instabilities which affect the timing of mainstream pulsars. This is frequently referred to as "timing" or "red" noise, and can be detrimental to long-term timing precision (e.g., Arzoumanian et al. 1994; Verbiest et al. 2009b; van Haasteren et al. 2009). For these reasons, in addition to their $2-3$ orders of magnitude shorter rotation rate, MSPs are thus the pulsars of choice when the highest timing precision is required, as is the case for the pursuit of gravitational wave (GW) detection.

Pulsar timing has been very successful for the measurement of pulsar properties, and the study of the environments around NSs. Perhaps its most celebrated results, however, are those that have tested general relativity (GR) and other theories of gravity.

\subsection{Testing gravitational theories with pulsars}

The very first test of GR using pulsar observations involved timing of the first-discovered binary pulsar, PSR B1913+16. Through high-precision timing of this double-neutron-star system, the measured decay of its orbit was found to agree spectacularly well with the orbital energy loss due to quadrupolar GW radiation from that system, as predicted by GR (Hulse \& Taylor 1975; Taylor \& Weisberg 1989). More recently, observations of the double-pulsar system PSR J0737-3039A/B, in which both NSs are observed as radio pulsars, have provided the most stringent test to date of GR in the strong-field regime (Kramer et al. 2006). In addition, several pulsars have been, and continue to be, used to test various aspects of GR and other theories of gravity. These include, for example, probing Strong Equivalence Principle violation by setting limits on phenomena predicted to exist by alternative theories of gravity. Among these are gravitational dipole radiation, secular evolution of binary parameters due to preferred frame effects, and a variation in Newton's gravitational constant (e.g., Nordtvedt 1990; Damour \& Taylor 1991; Wex 2000; Arzoumanian 2003; Verbiest et al. 2008; Lazaridis et al. 2009). 


\subsection{Direct detection of gravitational waves}

Although some of the tests described in the preceding subsection compare predictions of GR and other theories of gravity to pulsar observations in order to infer the existence of GW radiation as prescribed by GR, they fall short of being able to claim a direct detection of GWs. Pulsars can, however, be used to directly detect a stochastic GW background (GWB), presumably produced by supermassive black hole binaries at the centers of distant galaxies (Rajagopal \& Romani 1995; Jaffe \& Backer 2003) or perhaps by exotic phenomena such as cosmic strings (e.g., Damour \& Vilenkin 2005). This is done by using an ensemble of pulsars as arms of a very large GW detector. The perturbing effect of a GWB passing by the Earth would be correlated amongst observations of all pulsars. This would manifest itself as a distinct signature in the timing residuals, once all model parameters particular to the pulsar system were properly accounted for. The cross-correlation of these residuals is predicted to show a distinct dependence on the separations between the various pulsars observed, and so a distribution of pulsars evenly spread over as much of the sky as possible is desired for such a study (e.g., Detweiler 1979; Hellings \& Downs 1983).

A distribution of sources in a "pulsar timing array" (PTA) thus provides a unique laboratory for performing this experiment. The PTA experiment is sensitive to GWs with frequencies of $\sim 10^{-9}$ $\mathrm{Hz}$, since the limiting frequency corresponds to the timespan of the data sets used, typically $1-10$ years in length. Such a detection will be complementary to those that will be made by terrestrial and space-based interferometers such as LIGO (Abramovici et al. 1992) and LISA (Danzmann 2000), which are most sensitive to wave frequencies of $\sim 10-10^{3} \mathrm{~Hz}$ and $\sim 10^{-4}-10^{-2} \mathrm{~Hz}$, respectively.

The expected correlated signal is very subtle, and its detection requires the highest-precision timing, with rms of timing residuals well below $1 \mu \mathrm{s}$. It is also crucial that the pulsars used for this undertaking are stable over long timescales (e.g., Verbiest et al. 2009b). Ideally, observations of 20-40 MSPs are required to be observed over 5-10 years, with consistent $\sim 100 \mathrm{~ns}$ rms for the resulting timing residuals, in order to be able to significantly distinguish a GWB signal in the measured cross-correlation spectrum (Jenet et al. 2005).

The direct detection of GWs using a PTA is certainly a very challenging task. There now exist three major research groups dedicated to this effort: The Parkes Pulsar Timing Array (PPTA) in Australia, which uses the Parkes radio telescope (Verbiest et al. 2009a); the North American Nanohertz Observatory for Gravitational Waves (NANOGrav) collaboration in North America, which takes its data from the Arecibo and Green Bank telescopes (Jenet et al. 2009); and the European Pulsar Timing Array (EPTA). These groups have begun to develop a partnership, with the goal of sharing resources in an International Pulsar Timing Array (IPTA) effort, which will be necessary to achieve the objective of GW detection (Hobbs et al. 2009).

\section{The European Pulsar Timing Array}

The EPTA is a multi-national European collaboration of pulsar astronomers with the common aim of performing and improving high-precision pulsar timing, principally for the purpose of detecting GWs. It is also the goal of the EPTA to discover new candidate PTA pulsars, and as such, EPTA members are involved in various international pulsar search programs. The EPTA consists 
of members from various institutions in France, Germany, Italy, the Netherlands, and the United Kingdom, with continued ties to research groups in other countries around the world.

\section{Telescopes}

The EPTA employs the data from five large-diameter radio telescopes, all of which have ongoing pulsar observing programs, including those focused specifically on collecting MSP data for the purposes of a PTA. The EPTA telescopes are: the 100-meter Effelsberg radio telescope located at Effelsberg in Germany; the 76-meter Lovell radio telescope, located at the Jodrell Bank Observatory in the United Kingdom; the 94-meter-diameter-equivalent Nançay radio telescope, located in central France; the $14 \times 25$-meter telescope array WSRT, operated by ASTRON in the Netherlands and the 64-meter Sardinia Radio Telescope (SRT) currently under contruction by the National Institute of Astrophysics (INAF; Istituto Nazionale di Astrofisica) in Italy.

The EPTA is distinct among PTA collaborations, in that it continues to provide valuable experience in combining data from several observatories. This will demonstrate how to effectively and simultaneously deal with these varied data sets (see, e.g. Janssen et al. 2008). This will be of great importance for a future combined effort of an IPTA collaboration that will combine the data and resources of the NANO-Grav and PPTA groups with those of the EPTA.

A summary of properties of the five telescopes and a comparison with the other PTA telescopes, can be found in Ferdman et al. (2010). For a direct comparison of the timing capabilities of the three PTA groups, see Hobbs et al. (2010).

\section{Current progress}

All the currently operational telescopes of the EPTA have observing programs that involve the regular monitoring of MSPs. The quality of the data, and timing precision obtained, is comparable with those of the other PTA collaborations. See Ferdman et al. (2010) for a sample of MSPs currently observed by the EPTA.

There is also an active effort to model the observed timing residuals into a measurement of a GWB amplitude that is constrained by the EPTA data. This involves a Bayesian statistical analysis, employing a Markov Chain Monte Carlo algorithm to sample the likelihood function of the GWB amplitude (van Haasteren et al., 2009). At the present time, the timing precision of the EPTA pulsars is insufficient to achieve a significant GWB detection. However, using this method, we obtain a preliminary $95 \%$ upper limit to the GWB amplitude of $1.9 \times 10^{-14} \mathrm{yr}^{1 / 2}$; the likelihood function derived from this analysis is shown in Figure 1. For a wave with a frequency of $1 \mathrm{yr}^{-1}$, this corresponds to a GW energy density $h^{2} \Omega_{G W}=2.1 \times 10^{-7}$ (van Haasteren et al. 2010, in prep.). With more data, this limit will continue to become further constrained.

\section{LEAP: The Large European Array for Pulsars}

The existing collaboration of EPTA member institutions, together with the close proximity of 100-meter-class radio telescopes from each other, as well as the already well-established pulsar observing programs at these telescopes, presents a unique opportunity to combine the signals from 


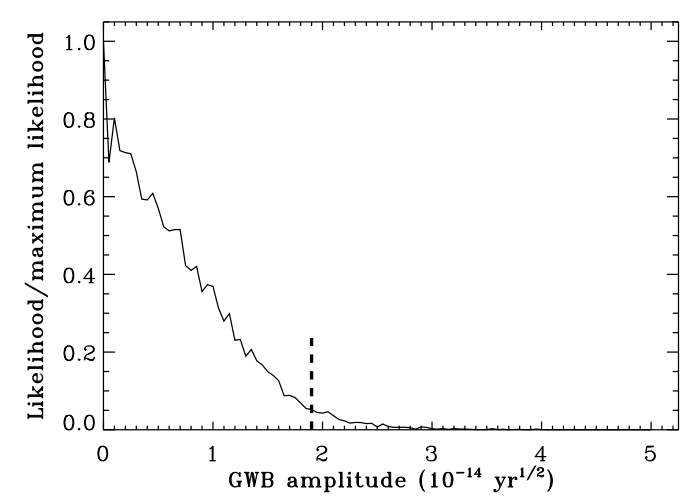

Figure 1: Likelihood function of the GWB amplitude derived from EPTA pulsar timing data, normalized so that the peak of the function has a value of 1.0. The dashed vertical line shows the $95 \%$ upper limit to the GW amplitude of $1.9 \times 10^{-14} \mathrm{yr}^{-1 / 2}$, corresponding to a GW energy density $h_{0}^{2} \Omega_{\mathrm{GW}}=2.1 \times 10^{-7}$ for a wave frequency of $1 \mathrm{yr}^{-1}$.

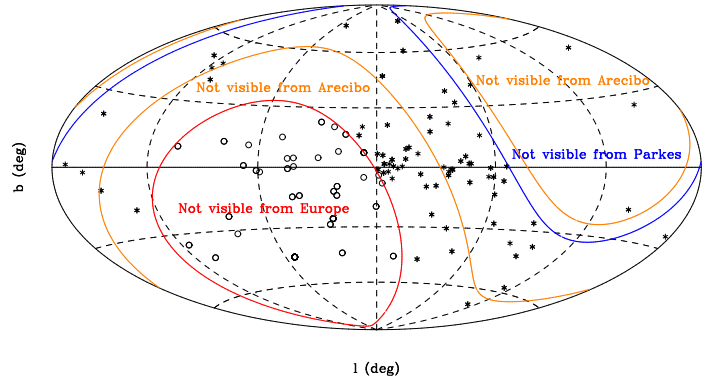

Figure 2: Comparison of the sky coverage limits of the Arecibo (dash-dot-dot-dot lines) and Parkes (strong dashed lines) telescopes with the future LEAP telescope array (strong solid line). Plotted are known MSPs, with those visible from Europe (and thus LEAP) labelled as stars. The central vertical and horizontal solid lines represent $0^{\circ}$ in 1 and $\mathrm{b}$, respectively. Divisions of $60^{\circ}$ in 1 and $30^{\circ}$ in $\mathrm{b}$ are represented by weak dashed lines.

the major European observatories in a coherent array. Such an endeavor will provide a significant boost to the quality of data used by the EPTA, enhancing its potential for detecting a GWB. This is the primary objective of the LEAP project, which is expected to see first light by the end of 2010.

LEAP will utilize techniques adapted from existing long-baseline arrays such as VLBI (e.g., Deller et al. 2007; Brisken et al. 2010) and MERLIN. However, unlike the imaging data that are obtained from those collaborations, LEAP will be designed specifically for taking pulsar timing data. The goal is to produce a time series from all the telescopes added coherently in phase, significantly increasing the sensitivity of pulsar observations. This will require techniques beyond the current state of the art in data-handling and processing. The "tied array" formed by the EPTA member telescopes will correspond to the equivalent of a single dish of approximately 194 meters in diameter or $29500 \mathrm{~m}^{2}$ of collecting area, comparable to that of the illuminated Arecibo radio telescope. This will substantially improve the timing results of the current timing array pulsars. Perhaps more importantly, the sensitivity of LEAP will allow for the precise study of MSPs that have otherwise been too weak for PTA study with other telescopes, including the individual member observatories of the EPTA. An important advantage that LEAP will have over the Arecibo telescope will be its much larger sky coverage. This will allow access to a greater number of currently-known pulsarsas well as those that will be discovered in the future-that can be used as part of a PTA. Figure 2 shows the distribution of known MSPs on the sky, and compares the ranges of sky visible from Arecibo, Parkes, and Europe (and thus LEAP). This will make LEAP the largest fully-steerable telescope in the world, and among the most sensitive, producing ideal data for high-precision pulsar timing and a major step forward in reaching PTA goals (e.g., Stappers et al. 2009). In addition to advancing the PTA effort to directly detect GW radiation, LEAP will provide a much-needed testbed for the future of radio observing techniques and precise pulsar timing with combined-signal telescope arrays. 


\section{Acknowledgements}

We are very grateful to all staff at the Effelsberg, Jodrell Bank, Nançay, and Westerbork radio telescopes for their help with observations used in this work. The LEAP project is funded through an European Reseach Council (ERC) grant to M. Kramer.

\section{References}

Abramovici, A., Althouse, W. E., Drever, R. W. P., et al. 1992, sci, 256, 325

Alpar, M. A., Cheng, A. F., Ruderman, M. A., \& Shaham, J. 1982, na, 300, 728

Arzoumanian, Z. 2003, Improved Bounds on Violation of the Strong Equivalence Principle

Arzoumanian, Z., Nice, D. J., Taylor, J. H., \& Thorsett, S. E. 1994, apj, 422, 671

Brisken, W. F., Macquart, J., Gao, J. J., et al. 2010, ApJ, 708, 232

Damour, T. \& Taylor, J. H. 1991, apj, 366, 501

Damour, T. \& Vilenkin, A. 2005, prevd, 71, 063510

Danzmann, K. 2000, asr, 25, 1129

Deller, A. T., Tingay, S. J., Bailes, M., \& West, C. 2007, PASP, 119,318

Detweiler, S. 1979, apj, 234, 1100

Ferdman, R. D., van Haasteren, R., Bassa, C. G., et al. 2010, Classical and Quantum Gravity, 27, 084014

Gold, T. 1968, na, 218, 731

Goldreich, P. \& Julian, W. H. 1969, apj, 157, 869

Hellings, R. W. \& Downs, G. S. 1983, apj1, 265, L39

Hobbs, G., Archibald, A., Arzoumanian, Z., et al. 2009, ArXiv e-prints

Hobbs, G., Archibald, A., Arzoumanian, Z., et al. 2010, Classical and Quantum Gravity, 27, 084013

Hulse, R. A. \& Taylor, J. H. 1975, apj, 195, L51

Jaffe, A. H. \& Backer, D. C. 2003, apj, 583, 616

Janssen, G. H., Stappers, B. W., Kramer, M., et al. 2008, A\&A, 490, 753
Jenet, F., Finn, L. S., Lazio, J., et al. 2009, ArXiv e-prints

Jenet, F. A., Hobbs, G. B., Lee, K. J., \& Manchester, R. N. 2005, apj1, 625, L123

Kramer, M., Stairs, I. H., Manchester, R. N., et al. 2006, nat, submitted

Lazaridis, K., Wex, N., Jessner, A., et al. 2009, MNRAS, 400,805

Lorimer, D. R. and Kramer, M. 2005, Handbook of Pulsar Astronomy (Cambridge University Press)

Nordtvedt, K. 1990, prl, 65, 953

Pacini, F. 1968, na, 219, 145

Radhakrishnan, V. \& Srinivasan, G. 1982, cursci, 51, 1096

Rajagopal, M. \& Romani, R. W. 1995, apj, 446, 543

Smarr, L. L. \& Blandford, R. 1976, apj, 207, 574

Srinivasan, G. \& van den Heuvel, E. P. J. 1982, aa, 108, 143

Stappers, B., Vlemmings, W., \& Kramer, M. 2009, in Proceedings of the 8th International e-VLBI Workshop. 22-26 June 2009. Madrid, Spain Published online at http://pos.sissa.it/cgibin/reader/conf.cgi?confid=82., p.20, 20-+

Sturrock, P. A. 1971, apj, 164, 529

Taylor, J. H. \& Weisberg, J. M. 1989, apj, 345, 434

van Haasteren, R., Levin, Y., McDonald, P., \& Lu, T. 2009, MNRAS, 395, 1005

Verbiest, J. P. W., Bailes, M., Bhat, N. D. R., et al. 2009a, ArXiv e-prints

Verbiest, J. P. W., Bailes, M., Coles, W. A., et al. 2009b, MNRAS, 400, 951

Verbiest, J. P. W., Bailes, M., van Straten, W., et al. 2008, ApJ, 679, 675

Wex, N. 2000, Small-eccentricity binary pulsars and relativistic gravity 